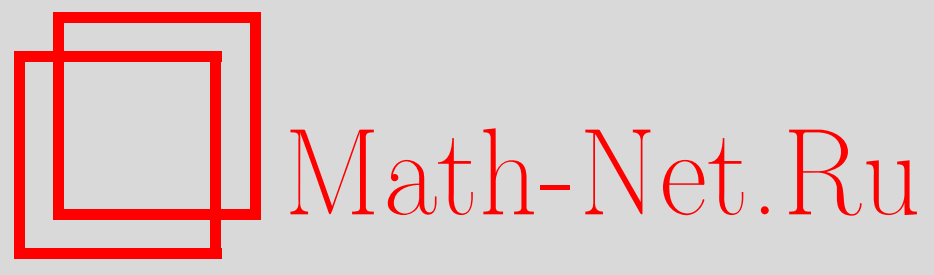

М. А. Власенко, Меры посещения для некоторых последовательностей случайных величин с убывающими нормировками, Теория вероятн. и ее примен., 2004, том 49, выпуск 1, 155-164

DOI: https://doi.org/10.4213/tvp241

Использование Общероссийского математического портала Math-Net.Ru подразумевает, что вы прочитали и согласны с пользовательским соглашением

http://www.mathnet.ru/rus/agreement

Параметры загрузки:

IP : 54.84 .234 .179

26 апреля 2023 г., 13:22:05

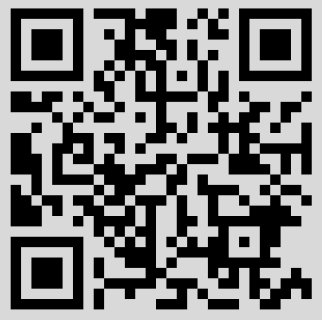


23. Baranov A.D. Isometric embeddings of the spaces $K_{\Theta}$ in the upper half-plane. - J. Math. Sci. (New York), 2001, v. 105, № 5, p. 2319-2329.

Поступила в редакцию 23.V.2002

(C) $2004 \mathrm{r}$.

ВЛАСЕНКО M. А.*

\title{
МЕРЫ ПОСЕЩЕНИЯ ДЛЯ НЕКОТОРЫХ ПОСЛЕДОВАТЕЛЬНОСТЕЙ СЛУЧАЙНЫХ ВЕЛИЧИН С УБЫВАЮЩИМИ НОРМИРОВКАМИ ${ }^{1)}$
}

\begin{abstract}
Для последовательности одинаково распределенных случайных величин $\left\{x_{n}, n \geqslant 1\right\}$, удовлетворяющей определенному условию перемешивания, исследуются локальные меры посешения последовательностей $\left\{x_{n} / a_{n}, n \geqslant 1\right\}$ с различными монотонно убывающими к 0 числовыми последовательностями $\left\{a_{n}, n \geqslant 1\right\}$. Получены достаточные условия того, что меры $\left\{\sum_{k=1}^{n} \mathbf{P}\left(x_{k} / a_{k}\right)^{-1}, n \geqslant 1\right\}$ являются локальными мерами посешения $\left\{x_{n} / a_{n}, n \geqslant 1\right\}$ с вероятностью 1 .
\end{abstract} ние.

Ключевые слова и фразы: локальные меры посешения, перемешива-

0. Введение. Пусть $\left\{x_{n}, n \geqslant 1\right\}$ - последовательность действительных чисел.

О п р е д е л е н и е 1. Считающими мерами последовательности $\left\{x_{n}, n \geqslant 1\right\}$ называется последовательность мер $\nu_{n}=\sum_{k=1}^{n} \delta_{x_{k}}, n \geqslant 1$.

Считающие меры содержат всю информацию о последовательности. Поэтому ее поведение на бесконечности может быть описано в терминах считаюших мер. Однако они устроены так же сложно, как и сама последовательность. Естественно попытаться заменить считаюшие меры более простой последовательностью мер, которая по-прежнему характеризовала бы $\left\{x_{n}, n \geqslant 1\right\}$ на бесконечности. Следуюшие определения были введены в [1] и [3].

О п р е д е л е н и е 2. Последовательность конечных на компактах мер $\left\{\lambda_{n}\right.$, $n \geqslant 1\}$ на борелевской $\sigma$-алгебре называется возрастаюшей, если $\lambda_{n+1}(\Delta) \geqslant \lambda_{n}(\Delta)$ для любого борелевского множества $\Delta$ при всех $n$ и $\lambda_{n}(\mathbf{R}) \rightarrow \infty$, когда $n \rightarrow \infty$.

Считающие меры последовательности действительных чисел являются примером возрастающей последовательности мер. Следующее определение обобщает понятие множества частичных пределов.

О п р е д е л е н и е 3. Для возрастающей последовательности мер $\left\{\lambda_{n}, n \geqslant 1\right\}$ множество $\mathfrak{M}_{\lambda}=\left\{x \in \mathbf{R} \mid \forall \varepsilon>0: \lambda_{n}((x-\varepsilon ; x+\varepsilon)) \longrightarrow \infty, n \rightarrow \infty\right\}$ называется ее предельным множеством, а элементы $\mathfrak{M}_{\lambda}$ - точками роста последовательности $\left\{\lambda_{n}, n \geqslant 1\right\}$.

Предельным множеством считающих мер $\left\{\nu_{n}, n \geqslant 1\right\}$ последовательности $\left\{x_{n}, n \geqslant 1\right\}$ является множество ее частичных пределов. Пусть $\Delta$ - окрестность некоторого частичного предела. Тогда $\left\{x_{n}, n \geqslant 1\right\}$ посетит $\Delta$ бесконечно много раз; возрастающая последовательность чисел $\left\{\nu_{n}(\Delta), n \geqslant 1\right\}$ показывает динамику появления членов последовательности в $\Delta$. Если нас не интересуют подробности этого процесса, то посешение множества $\Delta$ можно характеризовать произвольной последовательностью, эквивалентной $\nu_{n}(\Delta)$ при $n \rightarrow \infty$. Это и мотивирует следующее определение эквивалентности последовательностей мер.

О п р е д е л е н и е 4. Возрастающие последовательности мер $\left\{\lambda_{n}, n \geqslant 1\right\}$ и $\left\{\mu_{n}, n \geqslant 1\right\}$ называются локально равносходящимися, если $\mathfrak{M}:=\mathfrak{M}_{\lambda}=\mathfrak{M}_{\mu} \neq \varnothing$ и

* Институт математики НАН Украины, ул. Терешенковская, 3, 01601 Киев, Украина; e-mail: mariyka@imath.kiev.ua; url: http://masha.org.ua 
для любых открытых множеств $U_{1}$ и $U_{2}$ таких, что пересечение $U_{1}$ с $\mathfrak{M}$ непусто и $\bar{U}_{1}$ компактно и содержится в $U_{2}$, справедливо

$$
\varliminf_{n \rightarrow \infty} \frac{\lambda_{n}\left(U_{2}\right)}{\mu_{n}\left(U_{1}\right)} \geqslant 1 \quad \text { и } \quad \varliminf_{n \rightarrow \infty} \frac{\mu_{n}\left(U_{2}\right)}{\lambda_{n}\left(U_{1}\right)} \geqslant 1 .
$$

Обозначение: $\lambda_{n} \sim \mu_{n}$ при $n \rightarrow \infty$.

П р и м е $\mathrm{p} 1$. Пусть $\left\{x_{n}, n \geqslant 1\right\}$ - равномерно распределенная на отрезке $[0,1]$ последовательность (см. [5]), $\left\{\nu_{n}, n \geqslant 1\right\}$ - ее считающие меры, $\lambda$ - равномерное распределение на $[0,1]$. Покажем, что $\nu_{n} \sim n \lambda$ при $n \rightarrow \infty$. Действительно, предельным множеством обеих последовательностей мер является отрезок $[0,1]$. Если множества $U_{1}$ и $U_{2}$ такие, как в определении 4 , то в силу компактности $\bar{U}_{1}$ существует открытое множество $U$ с нулевой $\lambda$-мерой границы такое, что $\bar{U}_{1} \subset U \subset U_{2}$. Вследствие равномерной распределенности $\left\{x_{n}, n \geqslant 1\right\}$ на $[0,1]$ меры $\nu_{n} / n$ слабо сходятся к $\lambda$, когда $n \rightarrow \infty$. Тогда $\lim _{n \rightarrow \infty} \nu_{n}(U) /(n \lambda(U))=1$, и требования определения 4 для $U_{1}$ и $U_{2}$ выполнены.

Этот пример показывает, что асимптотику частоты посещения последовательностью окрестностей ее частичных пределов можно иногда охарактеризовать при помощи последовательности мер простого вида. В примере 1 окрестности всех частичных пределов посещаются одинаково часто.

П р и м е р 2. Пусть $\left\{x_{n}, n \geqslant 1\right\}$ - последовательность из примера 1. Определим $\left\{y_{n}, n \geqslant 1\right\}$ таким образом:

$$
y_{n}= \begin{cases}x_{n}, & n \neq 2^{l}, l \in \mathbf{N}, \\ 2-\frac{1}{n}, & n=2^{l}, l \in \mathbf{N} .\end{cases}
$$

Множеством частичных пределов последовательности $\left\{y_{n}, n \geqslant 1\right\}$ является $[0,1] \cup\{2\}$, причем для любой окрестности точки 2 , не пересекающейся с $[0,1]$, при больших $n$ приблизительно $\log _{2} n$ среди $n$ первых членов последовательности посещает эту окрестность.

О п р е д е л е н и е 5. Произвольная возрастающая последовательность мер, локально равносходящаяся со считающими мерами последовательности $\left\{x_{n}, n \geqslant 1\right\}$, называется последовательностью локальных мер посещения $\left\{x_{n}, n \geqslant 1\right\}$.

Так, в примере $2\left\{\lambda_{n}=n \lambda+\left(\log _{2} n\right) \delta_{2}, n \geqslant 1\right\}$ - локальные меры посещения $\left\{y_{n}, n \geqslant 1\right\}$.

Предложенное описание поведения числовых последовательностей оказывается очень выгодным в приложении к случайным последовательностям. Например, асимптотика посешения окрестностей частичных пределов, как и само множество частичных пределов, для произвольной последовательности $\left\{x_{n}, n \geqslant 1\right\}$ независимых случайных величин детерминированы: неслучайная последовательность мер $\left\{\sum_{k=1}^{n} \mathbf{P} x_{k}^{-1}, n \geqslant 1\right\}$ есть ее локальные меры посешения с вероятностью 1 (см. [1], [2]). Здесь $\mathbf{P}$ обозначает вероятность, т.е. меру на вероятностном пространстве, где определены соответствуюшие случайные величины. Для зависимых случайных величин этот результат также верен в случае, когда последовательность стационарна и удовлетворяет определенным условиям перемешивания (теорема 2.2 в [3]). Аналогичные теоремы доказаны для схем линейных (см. [1]) и нелинейных (см. [2]) итераций.

В настоящей работе для последовательности случайных величин $\left\{x_{n}, n \geqslant 1\right\}$ исследуются локальные меры посешения $\left\{x_{n} / a_{n}, n \geqslant 1\right\}$ с различными монотонно убывающими к 0 числовыми последовательностями $\left\{a_{n}, n \geqslant 1\right\}$. Эту задачу можно понимать как выявление характера сходимости к 0 подпоследовательностей исходной последовательности $\left\{x_{n}, n \geqslant 1\right\}$.

1. Основные результаты. В п. 2 будет предложено достаточное условие для того, чтобы последовательность $\left\{x_{n} / a_{n}, n \geqslant 1\right\}$ имела локальные меры посешения $\left\{\sum_{k=1}^{n} \mathbf{P}\left(x_{k} / a_{k}\right)^{-1}, n \geqslant 1\right\}$ с вероятностью 1 (теорема 3 ). Применяя его, мы получим следуюшие результаты.

Теорема 1. Пусть $\left\{x_{n}, n \geqslant 1\right\}$ - гауссовские случайные величины со средним 0 u дисперсией $1, \sup _{k \neq n}\left|\mathbf{E} x_{k} x_{n}\right|<1 u \sup _{n}\left(\sum_{k=1}^{n}\left|\mathbf{E} x_{k} x_{n}\right|\right)<\infty$. Тогда $\partial \Omega s$ любого а $\in(0,1)$ последовательность $\left\{n^{a} x_{n}, n \geqslant 1\right\}$ имеет локальные меры посещения $\left\{n^{1-a}((1-a) \sqrt{2 \pi})^{-1} \lambda, n \geqslant 1\right\}$ с вероятностью 1 , где $\lambda-$ мера Лебега. 
Рассмотрим пример использования этого результата.

П р и м е р 3 (линейные итерации с нарастающим шумом). Пусть $\left\{\xi_{n}, n \geqslant 1\right\}-$ независимые гауссовские случайные величины со средним 0 и дисперсией $1, \gamma \in(0,1)$ и $a \in(0,1)$. Последовательность $\left\{x_{n}, n \geqslant 1\right\}$, построенная по схеме $x_{0}=0$, $x_{n}=\gamma x_{n-1}+n^{a} \xi_{n}$ при $n \geqslant 1$, имеет с вероятностью 1 локальные меры посещения $\left\{n^{1-a} \sqrt{1-\gamma^{2}}((1-a) \sqrt{2 \pi})^{-1} \lambda, n \geqslant 1\right\}$.

Докажем это. Имеем $x_{n}=\sum_{k=1}^{n} \gamma^{n-k} k^{a} \xi_{k}$. Обозначим $a_{n}^{2}=\mathbf{E} x_{n}^{2}=$ $\sum_{k=1}^{n} \gamma^{2(n-k)} k^{2 a}$ и $y_{n}=x_{n} / a_{n}$ для всех $n$. Сначала покажем, что сушествует натуральное $N$ такое, что $\left\{y_{n}, n \geqslant N\right\}$ удовлетворяет условиям теоремы 1 .

Мы будем часто пользоваться следующей леммой из анализа. Пусть последовательности действительных чисел $\left\{z_{n}, n \geqslant 1\right\}$ и $\left\{v_{n}, n \geqslant 1\right\}$ таковы, что $\left\{v_{n}, n \geqslant 1\right\}$ строго монотонно возрастает к $+\infty$ и существует конечный или бесконечный $\lim _{n \rightarrow \infty}\left(z_{n}-z_{n-1}\right) /\left(v_{n}-v_{n-1}\right)$. Тогда $\lim _{n \rightarrow \infty} z_{n} / v_{n}=\lim _{n \rightarrow \infty}\left(z_{n}-z_{n-1}\right) /\left(v_{n}-\right.$ $\left.v_{n-1}\right)$. Это утверждение носит название теоремы Штольца (см. [6]).

По теореме Штольца

$$
\lim _{n \rightarrow \infty} \frac{a_{n}^{2}}{n^{2 a}}=\lim _{n \rightarrow \infty} \frac{\sum_{k=1}^{n} \gamma^{-2 k} k^{2 a}}{\gamma^{-2 n} n^{2 a}}=\lim _{n \rightarrow \infty} \frac{\gamma^{-2 n} n^{2 a}}{\gamma^{-2 n} n^{2 a}-\gamma^{-2(n-1)}(n-1)^{2 a}}=\frac{1}{1-\gamma^{2}} .
$$

Зафиксируем произвольное $\beta \in(\gamma, 1)$. Тогда найдется натуральное $N$ такое, что $a_{n} / a_{n+1}<\beta / \gamma$ при всех $n \geqslant N$. Значит, если $n>m \geqslant N$, то

$$
\mathbf{E} y_{m} y_{n}=\frac{a_{m}}{a_{n}} \gamma^{n-m}=\left(\frac{a_{m}}{a_{m+1}} \gamma\right)\left(\frac{a_{m+1}}{a_{m+2}} \gamma\right) \cdots\left(\frac{a_{n-1}}{a_{n}} \gamma\right)<\beta^{n-m} \leqslant \beta,
$$

и условие $\sup _{n \neq m}\left|\mathbf{E} y_{m} y_{n}\right| \leqslant \beta$ выполняется для $\left\{y_{n}, n \geqslant N\right\}$. Выполнение второго условия теоремы 1 следует из того, что по теореме Штольца

$$
\lim _{n \rightarrow \infty} \sum_{m=N}^{n}\left|\mathbf{E} y_{m} y_{n}\right|=\lim _{n \rightarrow \infty} \frac{\sum_{m=N}^{n} \gamma^{-m} a_{m}}{\gamma^{-n} a_{n}}=\lim _{n \rightarrow \infty} \frac{\gamma^{-n} a_{n}}{\gamma^{-n} a_{n}-\gamma^{-(n-1)} a_{n-1}}=\frac{1}{1-\gamma} .
$$

Из теоремы 1 следует, что последовательность $\left\{n^{a}\left(1-\gamma^{2}\right)^{-1 / 2} y_{n}, n \geqslant 1\right\}$ имеет локальные меры посещения $\left\{n^{1-a} \sqrt{1-\gamma^{2}}((1-a) \sqrt{2 \pi})^{-1} \lambda, n \geqslant 1\right\}$ с вероятностью 1 . Обозначим

$$
\alpha_{n}=\frac{n^{a}}{a_{n} \sqrt{1-\gamma^{2}}}, \quad \lambda_{n}=\frac{n^{1-a} \sqrt{1-\gamma^{2}}}{(1-a) \sqrt{2 \pi}} \lambda
$$

для всех $n$. Теперь покажем, что последовательности $\left\{x_{n}, n \geqslant 1\right\}$ и $\left\{\alpha_{n} x_{n}, n \geqslant 1\right\}$ имеют с вероятностью 1 одинаковые локальные меры посешения. Теорема 1 является следствием теоремы 3 из п. 2, при доказательстве которой будет построено множество полной вероятности $\Omega_{0}$ такое, что при каждом $\omega \in \Omega_{0}$ для каждого множества $\Delta$, являющегося конечным объединением полуоткрытых интервалов с концами в рациональных точках, верно $\lim _{n \rightarrow \infty} \sum_{k=1}^{n} \mathrm{I}_{\Delta}\left(\alpha_{k} x_{k}\right) / \lambda_{n}(\Delta)=1$. Пусть $\omega \in \Omega_{0}$, и пусть $U_{1}$ и $U_{2}$ - открытые множества из определения 4. Поскольку $\bar{U}_{1}-$ компакт, то найдется конечное объединение полуоткрытых интервалов с концами в рациональных точках $\Delta_{1}$ такое, что $U_{1} \subset \Delta_{1}$ и $\bar{\Delta}_{1} \subset U_{2}$. Обозначим $U\left(\Delta_{1}, \varepsilon\right)=\bigcup_{x \in \Delta_{1}}(x-\varepsilon, x+\varepsilon)$. Поскольку $\bar{\Delta}_{1}-$ компакт, то для некоторого $\varepsilon>0 U\left(\Delta_{1}, \varepsilon\right) \subset U_{2}$. И пусть конечное объединение полуоткрытых интервалов с концами в рациональных точках $\Delta_{2}$ таково, что $U\left(\Delta_{1}, \varepsilon\right) \subset \Delta_{2} \subset U_{2}$. В силу ограниченности $\Delta_{1}$ и того, что $U\left(\Delta_{1}, \varepsilon\right) \subset \Delta_{2}$ и $\alpha_{n} \rightarrow 1$, когда $n \rightarrow \infty$, сушествует натуральное $N=N(\varepsilon)$ такое, что для всех $n \geqslant N$ из $\left\{\alpha_{n} x_{n} \in \Delta_{1}\right\}$ следует $\left\{x_{n} \in \Delta_{2}\right\}$ и из $\left\{x_{n} \in \Delta_{1}\right\}$ следует $\left\{\alpha_{n} x_{n} \in \Delta_{2}\right\}$. Тогда

$$
\varliminf_{n \rightarrow \infty} \frac{\sum_{k=1}^{n} \mathrm{I}_{\Delta_{2}}\left(x_{k}\right)}{\sum_{k=1}^{n} \mathrm{I}_{\Delta_{1}}\left(\alpha_{k} x_{k}\right)} \geqslant 1 \quad \text { и } \quad \varliminf_{n \rightarrow \infty} \frac{\sum_{k=1}^{n} \mathrm{I}_{\Delta_{2}}\left(\alpha_{k} x_{k}\right)}{\sum_{k=1}^{n} \mathrm{I}_{\Delta_{1}}\left(x_{k}\right)} \geqslant 1 .
$$

(Знаменатель в первом соотношении не равен нулю начиная с некоторого $n$, поскольку $\lim _{n \rightarrow \infty} \sum_{k=1}^{n} \mathrm{I}_{\Delta_{1}}\left(\alpha_{k} x_{k}\right) / \lambda_{n}\left(\Delta_{1}\right)=1$. Тогда в силу первого соотношения не равен нулю начиная с некоторого $n$ и знаменатель во втором.) Получаем

$$
\varliminf_{n \rightarrow \infty} \frac{\sum_{k=1}^{n} I_{\Delta_{2}}\left(x_{k}\right)}{\lambda_{n}\left(\Delta_{1}\right)} \geqslant 1 \quad \text { и } \quad \varliminf_{n \rightarrow \infty} \frac{\lambda_{n}\left(\Delta_{2}\right)}{\sum_{k=1}^{n} I_{\Delta_{1}}\left(x_{k}\right)} \geqslant 1 .
$$


и требования определения 4 для $U_{1}$ и $U_{2}$ выполнены. Последовательность мер $\left\{\lambda_{n}\right.$, $n \geqslant 1\}$ является локально равносходящейся со считающими мерами последовательности $\left\{x_{n}, n \geqslant 1\right\}$ при каждом $\omega \in \Omega_{0}$, т.е. с вероятностью 1 .

Полученный результат можно интерпретировать следующим образом: существует множество полной вероятности $\Omega_{0}$ такое, что для каждого $\omega \in \Omega_{0}$ и каждой непрерывной финитной функции $f: \mathbf{R} \rightarrow \mathbf{R}$

$$
\frac{\sqrt{2 \pi}(1-a)}{\sqrt{1-\gamma^{2}} n^{1-a}} \sum_{k=1}^{n} f\left(x_{k}\right) \longrightarrow \int_{\mathbf{R}} f(x) d x, \quad n \rightarrow \infty .
$$

Действительно, в примере указано такое множество $\Omega_{0}$, что при каждом $\omega \in \Omega_{0}$ для каждого множества $\Delta$, являюшегося конечным объединением полуоткрытых интервалов с концами в рациональных точках, верно

$$
\lim _{n \rightarrow \infty} \frac{\sqrt{2 \pi}(1-a)}{\sqrt{1-\gamma^{2}} n^{1-a}} \sum_{k=1}^{n} \mathrm{I}_{\Delta}\left(x_{k}\right)=\lambda(\Delta) .
$$

Поскольку класс всех таких множеств $\Delta$ является определяющим слабую сходимость на любом компактном интервале действительной оси (см. [7]), отсюда следует наше утверждение для непрерывной финитной функции $f: \mathbf{R} \rightarrow \mathbf{R}$.

Теорема 2. Пусть $\left\{x_{n}, n \geqslant 1\right\}$ - одинаково распределенные случайные величины, распределение которых $\mu$ абсолютно непрерывно относительно меры Лебега $\lambda$ в некоторой окрестности 0. Причем существует конечный ненулевой предел

$$
\frac{d \mu}{d \lambda}(0):=\lim _{x \rightarrow 0} \frac{d \mu}{d \lambda}(x) .
$$

Пусть также выполнено условие на коэффичиент перемешивания

$$
\bar{\rho}:=\sup _{\substack{n \subset\{1, \ldots, n\} \\ S^{*}=\{1, \ldots, n\}-S}} \rho\left(\sigma\left(x_{k}, k \in S\right), \sigma\left(x_{m}, m \in S^{*}\right)\right)<1,
$$

где $\sigma\left(x_{k}, k \in S\right)$ обозначает $\sigma$-алгебру, порожденную соответствуюшими случайньми величинами, и для двух $\sigma$-алгебр $A$ и $B$

$$
\rho(A, B)=\sup _{f \in L_{2}(A), g \in L_{2}(B)}|\operatorname{corr}(f, g)| .
$$

Тогда для любого а $\in(0,1)$ последовательность $\left\{n^{a} x_{n}, n \geqslant 1\right\}$ имеет локальные меры посещения

$$
\left\{\frac{d \mu}{d \lambda}(0) n^{1-a}(1-a)^{-1} \lambda, n \geqslant 1\right\}
$$

с вероятностью 1.

2. Доказательства. Предлагаемый метод доказательства того, что последовательность $\left\{x_{n} / a_{n}, n \geqslant 1\right\}$ имеет с вероятностью 1 локальные меры посещения $\left\{\sum_{k=1}^{n} \mathbf{P}\left(x_{k} / a_{k}\right)^{-1}, n \geqslant 1\right\}$, использует следующие предположения:

(a) случайные величины $\left\{x_{n}, n \geqslant 1\right\}$ одинаково распределены (обозначим $\left.\mu=\mathbf{P} x_{1}^{-1}\right)$;

(b) $\left\{x_{n}, n \geqslant 1\right\}$ удовлетворяет следуюшему условию перемешивания для некоторых натуральных $p, m$ и положительных $\left\{q_{i}, r_{i}, 1 \leqslant i \leqslant m\right\}$, каждое из которых меньше $2 p$ : для каждого положительного $\gamma$ сушествуют константы $\left\{C_{i}(\gamma), 1 \leqslant i \leqslant m\right\}$ такие, что для любого вложенного в $[-\gamma, \gamma]$ интервала $\Delta=[\alpha, \beta)$ и для любого натурального $n$

$$
\mathbf{E}\left(\sum_{k=1}^{n}\left(\mathrm{I}_{\Delta}\left(x_{k}\right)-\mu(\Delta)\right)\right)^{2 p} \leqslant \sum_{i=1}^{m} C_{i}(\gamma) n^{q_{i}} \mu(\Delta)^{r_{i}}
$$

(c) последовательность $\left\{a_{n}, n \geqslant 1\right\}$ монотонно убывает к 0 ;

(d) сушествует монотонно возрастающая функция $f: \mathbf{R}_{+} \rightarrow \mathbf{R}_{+}$такая, что $f(x+$ 1) $/ f(x) \rightarrow 1$, когда $x \rightarrow \infty,[f(n+1)]>[f(n)]$ для любого $n([x]$ обозначает целую 
часть числа $x$, т.е. наибольшее целое число, не превосходящее $x$ ) и при всех $1 \leqslant i \leqslant m$ для произвольного числа $\alpha$

$$
\sum_{n=1}^{\infty} \frac{1}{f(n)^{2 p-q_{i}} \mu\left(\left[\alpha a_{[f(n+1)]}, \alpha a_{[f(n)]}\right)\right)^{2 p-r_{i}}}<\infty
$$

(для $\alpha<0$ границы отрезков нужно поменять местами).

Лемма 1. Пусть выполнень условия (a)-(d) $u \alpha>0$. Ecли $\sum_{k=1}^{\infty} \mu\left(\left[0, \alpha a_{k}\right)\right)=$ $\infty$, то с вероятностью 1 при $n \rightarrow \infty$

$$
\sum_{k=1}^{n} \mathrm{I}_{[0, \alpha)}\left(\frac{x_{k}}{a_{k}}\right) \sim \sum_{k=1}^{n} \mu\left(\left[0, \alpha a_{k}\right)\right) .
$$

(И аналогично - для $\alpha<0$.)

Д о к а з а т е л ь с т в о. Пусть $\left\{\nu_{n}, n \geqslant 1\right\}$ и $\left\{\mu_{n}, n \geqslant 1\right\}$ - считающие меры последовательностей $\left\{x_{n}, n \geqslant 1\right\}$ и $\left\{x_{n} / a_{n}, n \geqslant 1\right\}$ соответственно. Обозначим интервал $[0, \alpha)$ как $\Delta$ и для каждого натурального $n$ интервалы $\left[\alpha a_{n+1}, \alpha a_{n}\right]$ как $\Delta_{n}$. Тогда

$$
\mu_{n}(\Delta) \equiv \sum_{k=1}^{n} \mathrm{I}_{\Delta}\left(\frac{x_{k}}{a_{k}}\right)=\sum_{k=1}^{n} \nu_{k}\left(\Delta_{k}\right)+\nu_{n}\left(\bigcup_{k>n} \Delta_{k}\right)
$$

Введем также обозначения $D_{n}=\left[\alpha a_{[f(n+1)]}, \alpha a_{[f(n)]}\right)$ для каждого натурального $n$ (будем считать,-что $[f(1)]=1)$ и

$$
\begin{aligned}
\tilde{\mu}_{n}(\Delta) & =\sum_{k=1}^{n} \nu_{[f(k)]}\left(D_{k}\right)+\nu_{[f(n)]}\left(\bigcup_{k>n} D_{k}\right), \\
\tilde{\widetilde{\mu}}_{n}(\Delta) & =\sum_{k=1}^{n} \nu_{[f(k+1)]}\left(D_{k}\right)+\dot{\nu}_{[f(n+1)]}\left(\bigcup_{k>n} D_{k}\right), \\
\langle n\rangle & =\max \{m \in \mathbf{N} \mid[f(m)] \leqslant n\} .
\end{aligned}
$$

Очевидно, $\langle n\rangle \rightarrow \infty$, когда $n \rightarrow \infty$. Кроме того, $\tilde{\widetilde{\mu}}_{\langle n\rangle}(\Delta) \geqslant \mu_{n}(\Delta) \geqslant \tilde{\mu}_{\langle n\rangle}(\Delta)$, и если мы покажем, что с вероятностью единица $\widetilde{\mu}_{n}(\Delta) / \mathbf{E} \widetilde{\mu}_{n}(\Delta) \rightarrow 1$ и $\widetilde{\widetilde{\mu}}_{n}(\Delta) / \mathbf{E} \widetilde{\widetilde{\mu}}_{n}(\Delta) \rightarrow 1$, а также $\mathbf{E} \widetilde{\widetilde{\mu}}_{n}(\Delta) / \mathbf{E} \widetilde{\mu}_{n}(\Delta) \rightarrow 1, n \rightarrow \infty$, то получим отсюда, что с вероятностью единица $\mu_{n}(\Delta) / \mathbf{E} \mu_{n}(\Delta) \rightarrow 1$, когда $n \rightarrow \infty$, что и требуется доказать.

Из условия (b) следует, что

$$
\sum_{n=1}^{\infty} \mathbf{E}\left(\frac{\nu_{[f(n)]}\left(D_{n}\right)}{[f(n)] \mu\left(D_{n}\right)}-1\right)^{2 p}<\sum_{i=1}^{m} C_{i}\left(\alpha a_{1}\right) \sum_{n=1}^{\infty} \frac{1}{[f(n)]^{2 p-q_{i}} \mu\left(D_{n}\right)^{2 p-r_{i}}}
$$

где все ряды сходятся в силу условия (d). Тогда, применяя неравенство Чебышева и лемму Бореля-Кантелли, нетрудно установить, что с вероятностью 1 $\nu_{[f(n)]}\left(D_{n}\right) /\left([f(n)] \mu\left(D_{n}\right)\right) \rightarrow 1$, когда $n \rightarrow \infty$. По теореме Штольца с вероятностью $1 \sum_{k=1}^{n} \nu_{[f(k)]}\left(D_{k}\right) \sim \sum_{k=1}^{n}[f(k)] \mu\left(D_{k}\right)$ при $n \rightarrow \infty$. Из условия (d) следует, что $\sum_{n=1}^{\infty}\left(f(n)^{2 p-q_{i}} \mu\left(\bigcup_{k>n} D_{k}\right)^{2 p-r_{i}}\right)^{-1}<\infty$ для $1 \leqslant i \leqslant m$, откуда точно так же получаем, что $\nu_{[f(n)]}\left(\bigcup_{k>n} D_{k}\right) /\left([f(n)] \mu\left(\bigcup_{k>n} D_{k}\right)\right) \rightarrow 1$ с вероятностью 1 , когда $n \rightarrow \infty$. Тогда с вероятностью 1

$$
\frac{\widetilde{\mu}_{n}(\Delta)}{\mathbf{E} \widetilde{\mu}_{n}(\Delta)}=\frac{\sum_{k=1}^{n} \nu_{[f(k)]}\left(D_{k}\right)+\nu_{[f(n)]}\left(\bigcup_{k>n} D_{k}\right)}{\sum_{k=1}^{n}[f(k)] \mu\left(D_{k}\right)+[f(n)] \mu\left(\bigcup_{k>n} D_{k}\right)} \longrightarrow 1, \quad n \rightarrow \infty
$$

Из условия (d) следует

(e) $\sum_{n=1}^{\infty}\left(f(n+1)^{2 p-q_{i}} \mu\left(D_{n}\right)^{2 p-r_{i}}\right)^{-1}<\infty$ для $1 \leqslant i \leqslant m$.

Доказательство того, что $\widetilde{\widetilde{\mu}}_{n}(\Delta) / \mathbf{E} \widetilde{\widetilde{\mu}}_{n}(\Delta) \rightarrow 1$ с вероятностью единица, когда $n \rightarrow \infty$, проводится аналогично с помошью условий (b) и (е).

По теореме Штольца $\sum_{k=1}^{n}[f(k+1)] \mu\left(D_{k}\right) \sim \sum_{k=1}^{n}[f(k)] \mu\left(D_{k}\right)$, и тогда также $\mathbf{E} \widetilde{\widetilde{\mu}}_{n}(\Delta) / \mathbf{E} \tilde{\mu}_{n}(\Delta) \rightarrow 1, n \rightarrow \infty$. Лемма доказана.

Далее рассмотрим случай, когда распределение $\mu$ абсолютно непрерывно относительно меры Лебега $\lambda$ в окрестности 0, причем существует конечный ненулевой 
предел $\lim _{x \rightarrow 0} \frac{d \mu}{d \lambda}(x)$. Это условие уже позволяет оценить скорость сходимости к 0 подпоследовательностей $\left\{x_{n}, n \geqslant 1\right\}$. Ассимптотически они могут сходиться не быстрее любого сходящегося ряда. То есть для последовательностей $\left\{a_{n}, n \geqslant 1\right\}$ таких, что $\sum_{n=1}^{\infty} a_{n}<\infty$, последовательность $\left\{x_{n} / a_{n}, n \geqslant 1\right\}$ с вероятностью 1 не имеет предельных точек. Это следует из леммы Бореля-Кантелли, поскольку сейчас $\mathbf{P}\left\{\left|x_{n}\right| / a_{n}<C\right\} \sim C \frac{d \mu}{d \lambda}(0) a_{n}$ при $n \rightarrow \infty$.

Сделанное предположение позволяет обратить результат леммы 1 в утверждение о локальных мерах посещения (теорема 3). Но прежде отметим, что в этом случае условие (d) можно сформулировать проще:

$\left(\mathrm{d}^{\prime}\right)$ существует монотонно возрастающая функция $f: \mathbf{R}_{+} \rightarrow \mathbf{R}_{+}$такая, что $f(x+$ 1) $/ f(x) \rightarrow 1$, когда $x \rightarrow \infty,[f(n+1)]>[f(n)]$ для любого $n$ и

$$
\sum_{n=1}^{\infty} \frac{1}{f(n)^{2 p-q_{i}}\left(a_{[f(n)]}-a_{[f(n+1)]}\right)^{2 p-r_{i}}}<\infty \quad \text { для всех } \quad 1 \leqslant i \leqslant m .
$$

Теорема 3. Пусть $\left\{x_{n}, n \geqslant 1\right\}$ - одинаково распределенные случайные величины, распределение $\mu$ которых абсолютно непрерывно относительно меры Лебега $\lambda$ в окрестности 0, причем существует конечный ненулевой предел

$$
\frac{d \mu}{d \lambda}(0):=\lim _{x \rightarrow 0} \frac{d \mu}{d \lambda}(x),
$$

и выполнено (b). Пусть тажже $\left\{a_{n}, n \geqslant 1\right\}-$ монотонно убываюшая последовательность действительных чисел такая, что $\sum_{n=1}^{\infty} a_{n}=\infty$, и выполнено $\left(\mathrm{d}^{\prime}\right)$.

Тогда с вероятностью 1 последовательность $\left\{x_{n} / a_{n}, n \geqslant 1\right\}$ имеет локальные меры посещения

$$
\left\{\frac{d \mu}{d \lambda}(0)\left(\sum_{k=1}^{n} a_{k}\right) \lambda, n \geqslant 1\right\} .
$$

Док а за т е ль с т в о. Для $\alpha>0$

$$
\sum_{k=1}^{n} \mu\left(\left[0, \alpha a_{k}\right)\right) \sim \frac{d \mu}{d \lambda}(0)\left(\sum_{k=1}^{n} a_{k}\right) \lambda([0, \alpha)), \quad n \rightarrow \infty
$$

(поскольку $\sum_{n=1}^{\infty} a_{n}=\infty$ и потому применима теорема Штольца). То же самое имеет место для $\alpha<0$. Тогда мы можем применить лемму 1 , и с вероятностью 1

$$
\mu_{n}([0, \alpha)) \sim \frac{d \mu}{d \lambda}(0)\left(\sum_{k=1}^{n} a_{k}\right) \lambda([0, \alpha)), \quad n \rightarrow \infty,
$$

где $\left\{\mu_{n}, n \geqslant 1\right\}$ - считающие меры последовательности $\left\{x_{n} / a_{n}, n \geqslant 1\right\}$. Для произвольного интервала $[\alpha, \beta)$ имеем

$$
\begin{aligned}
\frac{\mu_{n}([\alpha, \beta))}{\frac{d \mu}{d \lambda}(0)\left(\sum_{k=1}^{n} a_{k}\right)} & =\frac{\mu_{n}([0, \beta))}{\frac{d \mu}{d \lambda}(0)\left(\sum_{k=1}^{n} a_{k}\right)}-\frac{\mu_{n}([0, \alpha))}{\frac{d \mu}{d \lambda}(0)\left(\sum_{k=1}^{n} a_{k}\right)} \\
& \longrightarrow \lambda([0, \beta))-\lambda([0, \alpha))=\lambda([\alpha, \beta))
\end{aligned}
$$

при $n \rightarrow \infty$. Точно так же для произвольного множества $\Delta$, являюшегося объединением конечного числа полуоткрытых интервалов,

$$
\mu_{n}(\Delta) \sim \frac{d \mu}{d \lambda}(0)\left(\sum_{k=1}^{n} a_{k}\right) \lambda(\Delta), \quad n \rightarrow \infty .
$$

Далее следуем доказательству теоремы 1 в [1]. Пусть $E-$ множество всех конечных объединений полуоткрытых интервалов с концами в рациональных точках. Для каждого $\Delta$ из $E$ положим

$$
\Omega_{\Delta}=\left\{\omega \mid \lim _{n \rightarrow \infty} \mu_{n}(\Delta)\left(\frac{d \mu}{d \lambda}(0)\left(\sum_{k=1}^{n} a_{k}\right) \lambda(\Delta)\right)^{-1}=1\right\} .
$$


По доказанному $\Omega_{\Delta}-$ множества полной вероятности. Положим $\Omega_{0}=\bigcap_{\Delta \in E} \Omega_{\Delta}$. Тогда, поскольку $E$ - счетное множество, $\Omega_{0}$ - множество полной вероятности. Мы покажем, что для всех $\omega$ из $\Omega_{0}$

$$
\mu_{n} \sim \frac{d \mu}{d \lambda}(0)\left(\sum_{k=1}^{n} a_{k}\right) \lambda \quad \text { при } \quad n \rightarrow \infty
$$

в смысле определения 4 .

Пусть $\omega \in \Omega_{0}$. Предельным множеством последовательности мер

$$
\left\{\frac{d \mu}{d \lambda}(0)\left(\sum_{k=1}^{n} a_{k}\right) \lambda, n \geqslant 1\right\}
$$

является $\mathbf{R}$. Для произвольных $x \in \mathbf{R}$ и $\varepsilon>0$ в $E$ найдется содержащий $x$ полуоткрытый интервал $\Delta$ такой, что $\Delta \subset(x-\varepsilon, x+\varepsilon)$. Тогда, по построению $\Omega_{0}$, $\lim _{n \rightarrow \infty} \mu_{n}((x-\varepsilon, x+\varepsilon)) \geqslant \lim _{n \rightarrow \infty} \mu_{n}(\Delta)=\infty$. То есть $\mathbf{R}$ является предельным множеством последовательности мер $\left\{\mu_{n}, n \geqslant 1\right\}$ при данном $\omega$. Пусть $U_{1}$ и $U_{2}$ - открытые множества из определения 4. Поскольку $\bar{U}_{1}$ - компакт, то в $E$ найдется множество $\Delta$ такое, что $\bar{U}_{1} \subset \Delta \subset U_{2}$. По построению $\Omega_{0}$ имеем $\lim _{n \rightarrow \infty} \mu_{n}(\Delta)\left(\frac{d \mu}{d \lambda}(0)\left(\sum_{k=1}^{n} a_{k}\right) \lambda(\Delta)\right)^{-1}=1$, и требования определения 4 для $U_{1}$ и $U_{2}$ выполнены. Теорема доказана.

В качестве примеров использования теоремы 3 докажем теоремы 1 и 2 .

Д ок аз а т ель с т в о т е о р е мы 1 . Пусть $\left\{x_{n}, n \geqslant 1\right\}$ - последовательность из условия теоремы. Зафиксируем $\gamma>0$, и пусть множество $\Delta \subset[-\gamma, \gamma]$. Для всех $n$ положим $\eta_{n}=\mathrm{I}_{\Delta}\left(x_{n}\right)-\sigma(\Delta)$, где $\sigma=P x_{1}^{-1}$. Тогда

$$
\mathbf{E} \eta_{k} \eta_{n}=\frac{1}{2 \pi} \iint_{\Delta \times \Delta} e^{\left(x^{2}+y^{2}\right) / 2} h\left(x, y, \mathbf{E} x_{k} x_{n}\right) d x d y
$$

где

$$
h(x, y, r)=\frac{1}{\sqrt{1-r^{2}}} \exp \left(-\frac{r^{2}\left(x^{2}+y^{2}\right)-2 r x y}{2\left(1-r^{2}\right)}\right)-1,
$$

и справедлива оценка $\mathbf{E} \eta_{k} \eta_{n} \leqslant \max _{(x, y) \in[-\gamma, \gamma]^{2}}\left|h\left(x, y, \mathbf{E} x_{k} x_{n}\right)\right| \sigma(\Delta)^{2}$. Можно показать, что для $|r|<1$ верно соотношение $\max _{(x, y) \in[-\gamma, \gamma]^{2}}|h(x, y, r)|=$ $\max (|h(-\gamma, \gamma, r)|,|h(\gamma, \gamma, r)|) \sim \gamma^{2}|r|$ при $r \rightarrow 0$. Тогда для любого $0<r_{0}<1$ существует константа $A\left(\gamma, r_{0}\right)$ такая, что если $|r| \leqslant r_{0}$, то $\max _{(x, y) \in[-\gamma, \gamma]^{2}}|h(x, y, r)| \leqslant$ $A\left(\gamma, r_{0}\right)|r|$. Получаем оценку $\mathbf{E} \eta_{k} \eta_{n} \leqslant A\left(\gamma, \sup _{l \neq m}\left|\mathbf{E} x_{l} x_{m}\right|\right) \sigma(\Delta)^{2}$ гри $k \neq n$. Тогда

$$
\begin{aligned}
\mathbf{E}\left(\sum_{k=1}^{n} \eta_{k}\right)^{2}= & \sum_{k=1}^{n} \mathbf{E} \eta_{k}^{2}+2 \sum_{k=2}^{n} \sum_{l=1}^{k-1} \mathbf{E} \eta_{l} \eta_{k} \leqslant n \sigma(\Delta) \\
& +2 n A\left(\gamma, \sup _{l \neq m}\left|\mathbf{E} x_{l} x_{m}\right|\right) \sup _{n}\left(\sum_{k=1}^{n}\left|\mathbf{E} x_{k} x_{n}\right|\right) \sigma(\Delta)^{2},
\end{aligned}
$$

откуда следует условие (b) с $p=1, m=1, q_{1}=1, r_{1}=1$.

Условие $\left(\mathrm{d}^{\prime}\right)$ для $a_{n}=n^{-a}$ при $0<a<1$ получается с функцией $f(x)=x^{m}$, где $m>2 /(1-a)$. Теорема доказана.

Д о к а з а т е л ь с т в о т е о р е м ы 2. Зафиксируем $\gamma>0$, и пусть множество $\Delta \subset[-\gamma, \gamma]$. Определим также $\eta_{n}=\mathrm{I}_{\Delta}\left(x_{n}\right)-\mu(\Delta)$ для всех $n$. Обозначим

$$
\bar{\rho}_{\eta}:=\sup _{\substack{s \subset\{1, \ldots, n\} \\ S^{*}=\{1, \ldots, n\}-S}} \rho\left(\sigma\left(\eta_{k}, k \in S\right), \sigma\left(\eta_{m}, m \in S^{*}\right)\right) .
$$

Очевидно, $\bar{\rho}_{\eta} \leqslant \bar{\rho}$, так как $\sigma$-алгебры, порожденные элементами $\left\{\eta_{n}, n \geqslant 1\right\}$, беднее порожденных элементами $\left\{x_{n}, n \geqslant 1\right\}$. Поскольку $\bar{\rho}_{\eta}<1$, то к последовательности $\left\{\eta_{n}, n \geqslant 1\right\}$ применима лемма 3 из [4], согласно которой сушествует константа $C\left(\bar{\rho}_{\eta}\right)$ такая, что

$$
\mathbf{E}\left(\sum_{k=1}^{n} \eta_{k}\right)^{4} \leqslant C\left(\bar{\rho}_{\eta}\right)\left(\sum_{k=1}^{n} \mathbf{E} \eta_{k}^{4}+\left(\sum_{k=1}^{n} \mathbf{E} \eta_{k}^{2}\right)^{2}\right) \leqslant C(\bar{\rho})\left(n \mu(\Delta)+n^{2} \mu(\Delta)^{2}\right)
$$


т.е. условие (b) выполнено с $p=2, m=2, q_{1}=r_{1}=1, q_{2}=r_{2}=2$.

Условие $\left(\mathrm{d}^{\prime}\right)$ для $a_{n}=n^{-a}$ при $0<a<1$ получается с функцией $f(x)=x^{m}$, где $m>2 /(1-a)$. Теорема доказана.

Приведем еше пример негауссовской последовательности, удовлетворяюшей условию перемешивания (b).

П р и м е р 4. Пусть $(\Omega, \mathscr{F}, \mathbf{P})=([0,1), \mathscr{B}([0,1)), \lambda)$, где $\lambda$ - мера Лебега. Положим $x_{n}(\omega)=\{\omega+\sqrt{n}\}$ для каждого $n$, где $\{\cdot\}$ обозначает дробную часть числа. Мы покажем, что последовательность $\left\{x_{n}, n \geqslant 1\right\}$ удовлетворяет условию (b) с $p=1$, $m=1, q_{1}=\frac{3}{2}, r_{1}=1$.

Пусть $\Delta=[\alpha, \beta) \subset[0,1)$ и для каждого $n: \eta_{n}=\mathrm{I}_{\Delta}\left(x_{n}\right)-\lambda(\Delta)$,

$$
\begin{aligned}
\operatorname{EI}_{\Delta}\left(x_{n}\right) \mathrm{I}_{\Delta}\left(x_{k}\right) & = \begin{cases}0, & \lambda(\Delta)<|\{\sqrt{n}\}-\{\sqrt{k}\}|, \\
\lambda(\Delta)-|\{\sqrt{n}\}-\{\sqrt{k}\}|, & \lambda(\Delta) \geqslant|\{\sqrt{n}\}-\{\sqrt{k}\}|,\end{cases} \\
& =(\lambda(\Delta)-|\{\sqrt{n}\}-\{\sqrt{k}\}|)_{+},
\end{aligned}
$$

$\mathrm{E} \eta_{n} \eta_{k}=(\lambda(\Delta)-|\{\sqrt{n}\}-\{\sqrt{k}\}|)_{+}-\lambda(\Delta)^{2}$.

Согласно [5], последовательность $\{\{\sqrt{n}\}, n \geqslant 1\}$ является равномерно распределенной, причем

$$
D_{N}:=\sup _{I \in \mathscr{B}([0,1))}\left|\frac{1}{N} \sum_{k=1}^{N} \mathrm{I}_{I}(\{\sqrt{k}\})-\lambda(I)\right|=O\left(\frac{1}{\sqrt{N}}\right) \quad \text { при } \quad N \rightarrow \infty .
$$

Пусть $C$ - такая константа, что $D_{N} \leqslant C / \sqrt{N}$ при всех $N$. Для всякой непрерывной функции $g$, имеющей на $[0,1]$ ограниченную вариацию $V(g)$, справедливо неравенство Коксмы (см. [5])

$$
\left|\frac{1}{N} \sum_{k=1}^{N} g(\{\sqrt{k}\})-\int_{0}^{1} g(x) d x\right| \leqslant V(g) D_{N} .
$$

Рассмотрим $g(x)=(\lambda(\Delta)-|\{\sqrt{n}\}-x|)_{+}$. Тогда $\int_{0}^{1} g(x) d x \leqslant \lambda(\Delta)^{2}, V(g) \leqslant 2 \lambda(\Delta)$.

$$
\int_{0}^{1} g(x) d x \leqslant \lambda(\Delta)^{2}, \quad V(g) \leqslant 2 \lambda(\Delta) .
$$

Из неравенства Коксмы с $N=n-1$ для такой функции $g$ получим оценку $\sum_{k=1}^{n-1}(\lambda(\Delta)-$ $|\{\sqrt{n}\}-\{\sqrt{k}\}|)_{+} \leqslant(n-1)\left(\lambda(\Delta)^{2}+2 \lambda(\Delta) C / \sqrt{n-1}\right)$.

Тогда $\sum_{k=1}^{n-1} \mathbf{E} \eta_{k} \eta_{n} \leqslant 2 \lambda(\Delta) C \sqrt{n-1}$ и существует такая константа $D$, что для всех $n$ и $\Delta$

$$
\begin{aligned}
\mathbf{E}\left(\sum_{k=1}^{n} \eta_{k}\right)^{2}= & \sum_{k=1}^{n} \mathbf{E} \eta_{k}^{2}+2 \sum_{k=2}^{n} \sum_{l=1}^{k-1} \mathbf{E} \eta_{l} \eta_{k} \leqslant n\left(\lambda(\Delta)-\lambda(\Delta)^{2}\right) \\
& +2 \sum_{k=2}^{n} 2 \lambda(\Delta) \sqrt{k-1} \leqslant D \lambda(\Delta) n^{3 / 2}
\end{aligned}
$$

что и требовалось доказать.

Пример 4 показывает, что условие перемешивания (b) для последовательности $\left\{x_{n}, n \geqslant 1\right\}$ не влечет стремления к 0 корреляции членов последовательности при росте разности их номеров, как это было в примере 3.

3. О классах возможных нормировок $\left\{a_{n}, n \geqslant 1\right\}$. Пусть $\left\{x_{n}, n \geqslant 1\right\}-$ одинаково распределенные случайные величины, распределение $\mu$ которых абсолютно непрерывно относительно меры Лебега $\lambda$ в некоторой окрестности 0 , причем существует конечный ненулевой предел $\lim _{x \rightarrow 0} \frac{d \mu}{d \lambda}(x)$. Рассмотренный нами метод нахождения локальных мер посещения последовательности $\left\{x_{n} / a_{n}, n \geqslant 1\right\}$ исходит из того, что для $\left\{x_{n}, n \geqslant 1\right\}$ выполняется определенное условие перемешивания (b), параметры которого и определяют класс монотонно убывающих к 0 последовательностей $\left\{a_{n}, n \geqslant 1\right\}$, к которым применимы наши рассуждения (условие $\left(\mathrm{d}^{\prime}\right)$ ). Однако пока мы не выяснили, каким образом можно проверить принадлежность последовательности $\left\{a_{n}, n \geqslant 1\right\}$ этому классу. А также, в связи со сделанным в предыдушем 
пункте замечанием о сходимости ряда $\sum_{n=1}^{\infty} a_{n}$, интересен ответ на вопрос: все ли последовательности $\left\{a_{n}, n \geqslant 1\right\}$ такие, что $\sum_{n=1}^{\infty} a_{n}=\infty$, принадлежат этому классу?

Чтобы избежать громоздких записей, будем считать, что класс последовательностей $\left\{a_{n}, n \geqslant 1\right\}$ определяется условием

$\left(\mathrm{d}^{\prime \prime}\right)$ существует монотонно возрастаюшая функция $f: \mathbf{R}_{+} \rightarrow \mathbf{R}_{+}$такая, что $f(x+$ 1) $/ f(x) \rightarrow 1$, когда $x \rightarrow \infty,[f(n+1)]>[f(n)]$ для любого $n$ и $\sum_{n=1}^{\infty}\left\{f(n)^{c}\left(a_{[f(n)]}-\right.\right.$ $\left.\left.a_{[f(n+1)]}\right)^{d}<\infty\right\}^{-1}$.

(Числа $c$ и $d$ положительны.)

Лемма 2. Для того чтобы последовательность $\left\{a_{n}=n^{-a} \ln ^{-b} n ; n \geqslant 1\right\}$ с а > 0 удовлетворяла условию $\left(\mathrm{d}^{\prime \prime}\right)$, необходимо и достаточно, чтобь

$$
\left[\begin{array}{l}
a<\frac{c}{d}, \\
a=\frac{c}{d}, \quad b<-\frac{1}{d} .
\end{array}\right.
$$

Доказательство. Н е об бо ди м о сть.

Обозначим $g(x)=$ $x^{-a} \ln ^{-b} x$. Из условия $\left(\mathrm{d}^{\prime \prime}\right)$ следует, что $\sum_{n=1}^{\infty}\left(f(n)^{c} g(f(n))^{d}\right)^{-1}<\infty$. Начиная с некоторого значения аргумента, функция $h(x)=\left(x^{c+1} g(x)^{d}\right)^{-1}=\left(\ln ^{b d} x\right) / x^{1+c-d a}$ монотонна. Если она монотонно возрастает, то монотонно возрастают члены ряда $\sum_{n=1}^{\infty}\left(f(n)^{c} g(f(n))^{d}\right)^{-1}$, что невозможно. Значит, $h(x)$ монотонно убывает. Из того, что $\sum_{n=1}^{\infty}\left(f(n)^{c} g(f(n))^{d}\right)^{-1}<\infty$, и условия на $f$ следует сходимость ряда $\sum_{n=1}^{\infty}(f(n+1)-f(n)) /\left(f(n)^{c+1} g(f(n))^{d}\right)$. Тогда $\int_{1}^{\infty} h(x) d x<\infty$, откуда следует, что или $1+c-d a>1$, или $1+c-d a=1, b d<-1$.

Д о с т а т о ч н о с т ь. Мы покажем, что $\left\{a_{n}, n \geqslant 1\right\}$ для некоторого $\alpha \in(0,1)$ удовлетворяет условию $\left(\mathrm{d}^{\prime \prime}\right)$ с $f(x)=e^{x^{\alpha}}$. Для этого сначала убедимся, что $\sum_{n=1}^{\infty}\left(f(n)^{c}\left|g^{\prime}(f(n))\right|^{d} f^{\prime}(n)^{d}\right)^{-1}<\infty$ для некоторого $\alpha \in(0,1)$. Имеем

$$
\begin{aligned}
& g^{\prime}(x)=-\left(\frac{a}{x^{a+1} \ln ^{b} x}+\frac{b}{x^{a+1} \ln ^{b+1} x}\right) \sim-\frac{a}{x^{a+1} \ln ^{b} x}, \quad x \rightarrow \infty, \\
& f^{\prime}(x)=\alpha x^{\alpha-1} e^{x^{\alpha}}
\end{aligned}
$$

и тогда при $n \rightarrow \infty$

$f^{c}(n)\left|g^{\prime}(f(n))\right|^{d} f^{\prime}(n)^{d} \sim e^{c n^{\alpha}} \frac{a^{d}}{e^{(a+1) d n^{\alpha}} n^{b d \alpha}} \alpha^{d} n^{d(\alpha-1)} e^{d n^{\alpha}}=(\alpha a)^{d} n^{d(\alpha-1-\alpha b)} e^{(c-a d) n^{\alpha}}$.

Действительно, при условии (1) такое $\alpha$ найдется.

Очевидно, что

$$
\sum_{n=1}^{\infty} \frac{1}{f(n)^{c}(g([f(n)])-g([f(n+1)]))^{d}}<\sum_{n=1}^{\infty} \frac{1}{f(n)^{c}(g(f(n))-g(f(n+1)-1))^{d}}
$$

а сходимость последнего ряда эквивалентна сходимости ряда $\sum_{n=1}^{\infty}\left(f(n)^{c}(g(f(n))-\right.$ $\left.g(f(n+1)))^{d}\right)^{-1}$. Действительно, для достаточно больших $n$

$$
\frac{g(f(n+1)-1)-g(f(n+1))}{g(f(n))-g(f(n+1))}<\frac{g^{\prime}(f(n+1)-1)}{g^{\prime}(f(n)) f^{\prime}(n+1)}<\frac{1}{f^{\prime}(n+1)},
$$

а $f^{\prime}(x) \rightarrow \infty$, когда $x \rightarrow \infty$. В силу монотонности $f, f^{\prime}$ и $g^{\prime}$

$$
\sum_{n=1}^{\infty} \frac{1}{f(n)^{c}(g(f(n))-g(f(n+1)))^{d}}<\sum_{n=1}^{\infty} \frac{1}{f(n)^{c}\left|g^{\prime}(f(n+1))\right|^{d}-f^{\prime}(n)^{d}} .
$$

Поскольку, как и для функции $f$, для ее производной справедливо $f^{\prime}(x+1) / f^{\prime}(x) \rightarrow 1$, когда $x \rightarrow \infty$, то сходимость последнего ряда следует из доказанного выше. Лемма доказана.

Тогда критерием принадлежности $\left\{a_{n}=n^{-a} \ln ^{-b} n ; n \geqslant 1\right\}$ классу, для которого выполняется $\left(\mathrm{d}^{\prime}\right)$, будет

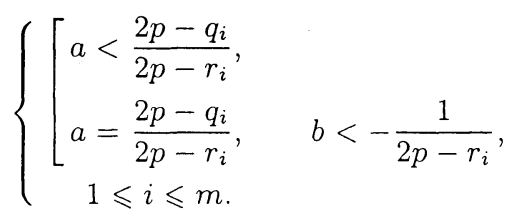


Так, в условии теоремы 1 можно было делить $\left\{x_{n}, n \geqslant 1\right\}$ на все последовательности вида $\left\{n^{-a} \ln ^{-b} n, n \geqslant 1\right\}$, где $a<1$, и на последовательности вида $\left\{n^{-1} \ln ^{b} n, n \geqslant 1\right\}$ с $b>1$. Для теоремы 2 - на $\left\{n^{-a} \ln ^{-b} n, n \geqslant 1\right\}$ с $a<1$ и на $\left\{n^{-1} \ln ^{b} n, n \geqslant 1\right\}$ с $b>\frac{1}{2}$. В примере 4 условия будут таковы: $a<\frac{1}{2}$ для $\left\{n^{-a} \ln ^{-b} n, n \geqslant 1\right\}$ или $b>1$ для $\left\{n^{-1 / 2} \ln ^{b} n, n \geqslant 1\right\}$.

Автор выражает благодарность Андрею Анатолиевичу Дороговцеву за постановку задачи и полезные советы при подпотовке работы.

\section{СПИСОК ЛИТЕРАТУРЫ}

1. Дороговцев A.A. Некоторые характеристики последовательностей итераций со случайными возмушениями. - Укр. матем. журн., 1996, т. 48, № 8, с. 1047-1063.

2. Дороговчев A. A. Меры посешения и эргодическая теорема для последовательности итераций со случайными возмущениями. - Укр. матем. журн., 1999, т. 51, № 1 , c. 123-127.

3. Dorogovtsev A.A., Denis'evskij N. A. Path-wise behavior of stationary sequences. Theory Stochastic Process., 1996, v. 2 (18), № 3-4, p. 17-26.

4. Bryc W., Smoleński W. Moment conditions for almost sure convergence of weakly correlated random variables. - Proc. Amer. Math. Soc., 1993, v. 119, № 2, p. 629635.

5. Кеŭперс Л., Нидеррейтер Г. Равномерное распределение последовательностей. M.: Наука, 1965, 408 c.

6. Дороговчев А.Я. Математический анализ. Ч. 1. Киев: Лыбидь, 1993.

7. Биллингсли П. Сходимость вероятностных мер. М.: Наука, 1977, 351 с.

Поступила в редакцию

9.II. 2000

Исправленный вариант

30.VII.2001

(c) 2004 г.

\section{ГРАНОВСКИЙ Б. Л.*, ЗЕЙФМАН А. И.** О НИЖНЕЙ ГРАНИЦЕ СПЕКТРА ДЛЯ НЕКОТОРЫХ МОДЕЛЕЙ СРЕДНЕГО ПОЛЯ ${ }^{1)}$}

Находится нижняя граница спектра инфинитезимальной матрицы для моделей среднего поля в случае, когда число взаимодействуюших частиц стремится к бесконечности.

Проводится также сравнение асимптотического поведения нижней границы спектра и спектральной лакуны, при этом находится класс моделей среднего поля, для которого границы спектра принимают экстремальные значения. Результаты получены с помошью метода, предложенного в 80-х годах вторым автором.

* Department of Mathematics, Technion-Israel Institute of Technology, Haifa, 32000 Israel; e-mail: mar18aa@techunix.technion.ac.il

** Вологодский госпедуниверситет и Вологодский научно-координационный центр ЦЭМИ РАН, ул. Орлова, 6, Вологда, Россия; e-mail: zai@uni-vologda.ac.ru

1) Работа первого автора поддержана Фондом содействия исследованиям Техниона. Работа над статьей начата во время визита второго автора в Технион в апреле 1997 г. при финансовой поддержке математического факультета Техниона. 